\title{
A conserved principle of glial organization in the paleocortex and neocortex
}

Short title: A conserved principle of glia organization in mammals

Antonio Pinto-Duarte ${ }^{1,4}$, Katherine Bogue ${ }^{2}$, Terrence J. Sejnowski ${ }^{1,5}$ and Shyam Srinivasan ${ }^{2,3,{ }^{*}}$

${ }^{1}$ Computational Neurobiology Laboratory, Salk Institute for Biological Studies; La Jolla, CA 92037

${ }^{2}$ Molecular Neurobiology Laboratory, Salk Institute for Biological Studies; La Jolla, CA 92037

${ }^{3}$ Kavli Institute for Brain and Mind, University of California, San Diego, CA 92093, USA

${ }^{4}$ Institute for Neural Computation, University of California, San Diego; CA 92093

${ }^{5}$ Division of Biological Sciences, University of California, San Diego; CA 92161

Keywords: Astrocyte, cortical thickness, scaling, paleocortex, brain organization.

*Corresponding author: Shyam Srinivasan (shyam@snl.salk.edu) 


\begin{abstract}
Neocortical and paleocortical neurons perform complex computations that depend on the architecture of the circuits and the environment in which they are embedded. The environment is largely governed by glial cells, and previous work has shown that the number of glial cells under a square mm depends on thickness of the neocortex, irrespective of the species. We wondered if this might be a principle of glial organization. To this end, we investigated if the paleocortex, which differs from the neocortex in its architecture, nevertheless obeys the same principle. We quantified the number of glial cells in the anterior piriform cortex, a paleocortical circuit subserving olfaction, across six mammalian species, using stereology and light microscopy. We found two orderly relationships across species. First, just like the neocortex, the number of glial cells per $\mathrm{mm}^{2}$ increased proportionally with cortical thickness, although the paleocortex had a higher density of glial cells. Second, the number of glia was proportional to piriform cortex size, indicating a conserved principle of organization across species. In conclusion, our findings show that glial cells are organized similarly in neocortex and paleocortex, although at different densities, likely reflecting local computational demands.
\end{abstract}




\section{Introduction}

While numerous studies have carefully examined scaling relationships and organizational principles of neurons in different brain regions (1-4), very few have examined corresponding relationships for glia. These cells are no less important, as they control the environment in which neurons perform complex computations. In the mature central nervous system, there are three major classes of glial cells: oligodendrocytes, which are responsible for the myelination of nerve fibers; microglia, which are involved in the immune response; and the most abundant, astrocytes, which play a wide range of roles, as discussed below. Previous studies have shown that the neocortices of animals with larger brains, such as primates, contain more glial cells per neuron (5), raising the question of the organizational principle that might account for such observations. A recent study addressed this issue by showing that the number of glial cells under a square $\mathrm{mm}$ of neocortical surface depends on the thickness of the neocortex (6). Thus, the increase in glial cells in larger brains mostly reflects an increase in the thickness of the neocortical surface rather than a "phylogenetic advance".

The relationship between neocortical thickness and number of glia likely results from the territorial disposition of astrocytes (7-9). Astrocytes are particularly important, with functions ranging from the regulation of the endothelium (10), $\mathrm{K}^{+}$buffering (11), glutamate clearance (1215), metabolism (16), fine-tuning of synaptic transmission $(17,18)$, synaptic plasticity $(19,20)$, and synchronization of the neural network (21-23). Notably, a similar association between neurons and astrocytes is also apparent in structures that are phylogenetically older and structurally different compared to the neocortex (24), such as the anterior segment of the piriform cortex, the largest region within the paleocortex. This raises the possibility that there might be a conserved principle for describing glial organization across phylogenetically different brain structures.

To answer these questions, we examined the anterior piriform cortex (Fig. 1A) of six mammalian species: mouse, rat, guinea pig, opossum, ferret, and cat, and quantified the 
number of glial cells contained under $1 \mathrm{~mm}^{2}$ of cortical surface. We made the following two discoveries: the number of glia below a square $\mathrm{mm}$ of piriform cortex surface is proportional to its thickness, and the total number of glia is proportional to the volume of piriform cortex, i.e. glial volume density is invariant. These two orderly relationships show evidence that glia follow the same organizational principle in the piriform cortex across species. Our evidence, combined with previous data (6), shows that glial cells follow a common principle of organization in the neocortex and paleocortex: the glial volume density is invariant across species (and brain sizes). It is possible that that such a relationship could extend to other brain regions, albeit at differing densities (as we will later discuss), to accommodate the differing computational demands of each region.

\section{Results}

The piriform cortex abuts the lateral olfactory tract, and is ventral to the rhinal sulcus and caudal to the olfactory bulb (Fig. 1A). It differs from the neocortex in three critical and interlinked ways. First, in terms of morphology, the piriform cortex is a trilaminar structure unlike the sixlayered neocortex. It comprises a sparsely (in terms of cells) populated layer I, a densely packed layer II, and an intermediately dense layer III (25). Second, while neocortical circuits largely receive topographic inputs, inputs into the piriform seem randomly distributed with bulbar axons synapsing with piriform neurons that are spread over the entire cortex (26). Third, they differ functionally too. In neocortical circuits, peripheral sensory inputs- for instance, retinal input into the visual cortex- evoke topographic activation patterns (27). In contrast, olfactory input evokes activity in a neuronal ensemble distributed throughout the piriform, reflecting the seemingly distributed input connectivity $(28,29)$.

Evidence from anatomical, physiological, and functional studies has suggested that the piriform cortex can be subdivided into two regions, the posterior piriform cortex (PPC), and the 
anterior piriform cortex (APC) (30). In this report, we focused on the APC, and from here on when we refer to the piriform cortex, we mean the APC.

To test for common principles of glial organization, we needed to estimate two quantities: first, the surface areas of piriform cortices; second, the number of glial cells underneath a square $\mathrm{mm}$ of piriform cortex. Together, these quantities would help determine if glia follow an orderly relationship across brain sizes.

\section{The surface area density of glial cells is proportional to the thickness of the}

\section{piriform cortex, like the neocortex}

We started by estimating the size of the piriform cortex across species. As shown in Fig S1A (Table 1), as brain size increases, the surface area of the piriform cortex also increases. Next, we estimated the number of glial cells underneath 1 square $\mathrm{mm}$ of piriform cortex across the six species, as shown in Fig. S1B (Table 1, and Table S1 for individual animal counts). From these two estimates we were able to estimate the number of glial cells in the piriform cortex for each of the six species as shown in Figure S1D. As the surface area of the piriform cortex increases, the number of glia increase proportionately $\left(R^{2}=0.987\right)$.
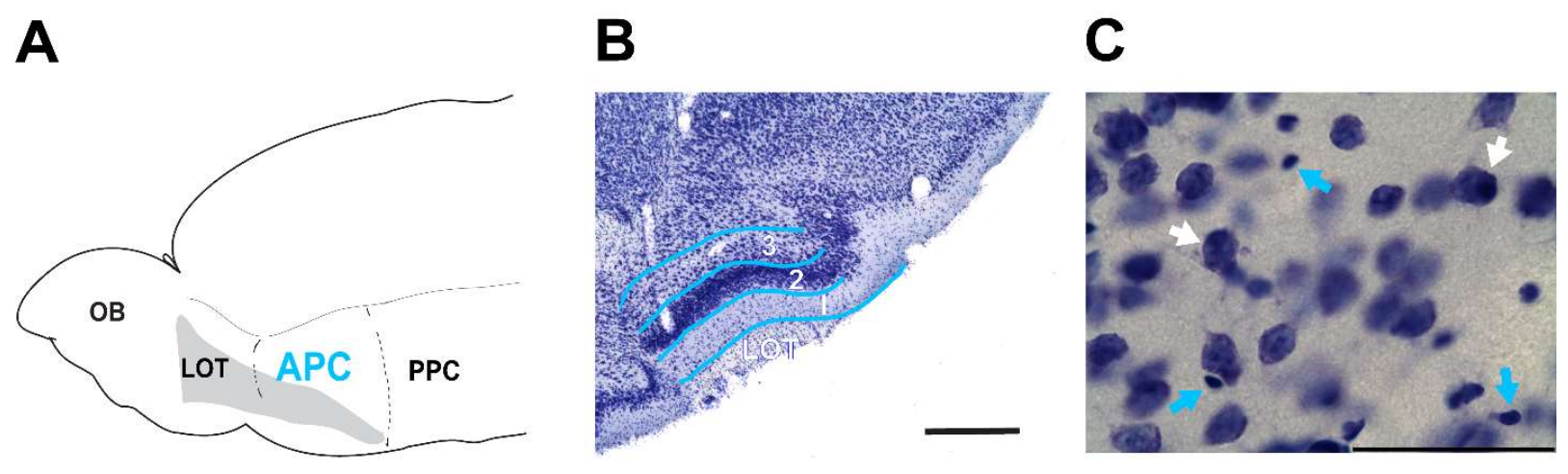

Figure 1: The piriform cortex of the mouse brain. (A) A side-view schematic of the mouse brain showing the anterior piriform cortex $(A P C)$, the olfactory bulb (OB), the lateral olfactory tract (LOT), and the posterior piriform cortex (PPC). (B) A representative Nissl-stained section from the mouse piriform cortex. The colored lines highlight the limits of each one of the three 
layers (numbered 1-3) within the APC. Scale bar: $500 \mu \mathrm{m}$. (C) Glia and neurons were identified according to well-established criteria (see methods), namely the absence (blue arrows, glia) or the presence of a visible nucleolus (white arrows, neurons). Image depicts a visual field from layer 3. Scale bar: $50 \mu \mathrm{m}$.

In (6), researchers compared changes in cortical thickness versus changes in surface area densities of glial cells, and showed that glial volume densities were invariant. To perform a similar comparison, we estimated the thickness of the piriform cortex across the six species, as shown in Fig. S1C (Table 1, and Table S1 for individual animal counts). From these estimates, we were able to determine the relationship between the number of glial cells underneath a square $\mathrm{mm}$ and the thickness of the cortex (Fig. 2A). Across species, the number of glial cells underneath a square $\mathrm{mm}$ is proportional to the thickness of the cortex. Thus, just like in the neocortex, as the piriform cortex becomes thicker, the volume of neuropil and number of glial cells increase together in a way that keeps glial volume density constant.

The data were fit with a regression line that had an associated $R^{2}$ value (coefficient of determination) of 0.89 and provided an opportunity to calculate the volume density of glial cells. The line is of the form $\mathrm{G}_{\mathrm{sad}}=\mathrm{N}_{0}+m^{*} \mathrm{PC} \mathrm{X}_{\text {thickness, }}$ where $\mathrm{G}_{\text {sad }}$ is the surface density in $\mathrm{glia} / \mathrm{mm}^{2}$, $\mathrm{N}_{0}$ is an additive constant (intercept) in units of $\mathrm{glia} / \mathrm{mm}^{2}, m$ is the slope in units of $\mathrm{glia} / \mathrm{mm}^{3}$, and $P C x_{\text {thickness }}$ is the thickness of the piriform cortex in $\mathrm{mm}$. Here $m$ is the volume density of glial cells $\left(G_{v}\right)$ in $\mathrm{glia} / \mathrm{mm}^{3}$ and is $55,000 \mathrm{glia} / \mathrm{mm}^{3}$ (95\% Confidence Interval, 24,000-99,000).

\section{The volume density of glia is invariant across species}

We also used our estimates or measurements in two other ways to verify that glial volume density is invariant across species. First, we directly estimated the average glial volume density from our measurements of cortical columns across the six species, and found it to be $45,000 \pm 2,600 \mathrm{glia} / \mathrm{mm}^{3}$ (mean \pm sem, Table S3), which is not significantly different from the estimate obtained from Fig. 2A. 

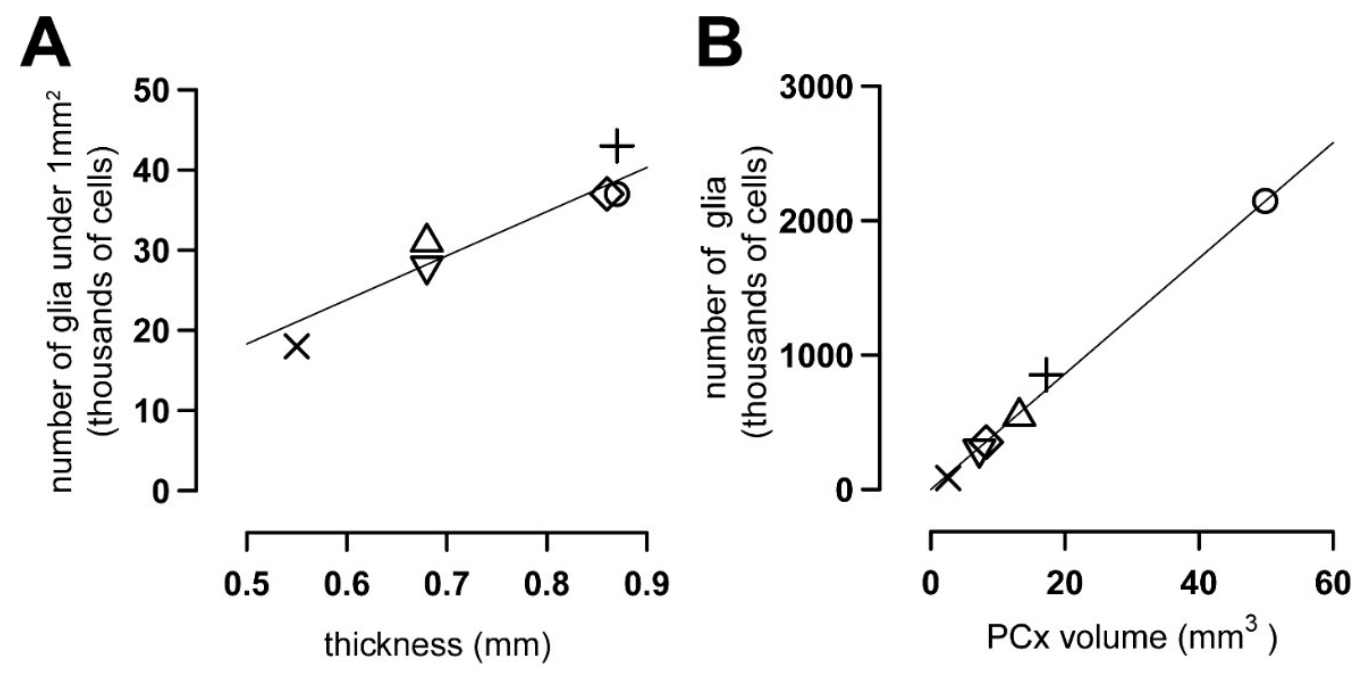

Figure 2: The thickness of the piriform cortex determines the number of glia cells, irrespectively of the species examined.

The number of glial cells was

plotted for six species of mammals: mouse $(\times)$, rat $(\nabla)$, ferret $(\Delta)$, opossum $(\diamond)$, cat (o), guinea pig (+). (A) Scatter plot of the number of glial cells under $1 \mathrm{~mm}^{2}$ of cortical surface against APC thickness. Note that the number of glia positively correlated with APC thickness, but this did not depend upon the species, as shown by a significant amount of overlap between symbols. The regression line $\left(R^{2}=0.89\right.$, Coefficient of determination) is described by the equation: $G_{s a d}=-9+$ $55^{\star} P C x_{\text {thickness, }}$ where $G_{\text {sad }}$ is number of glia (in thousands) under $1 \mathrm{~mm}^{2}$ and $P C x_{\text {thickness }}$ is thickness of the piriform cortex in $\mathrm{mm}$. The slope is $55,000 \mathrm{glia} / \mathrm{mm}^{3}$ (95\% confidence interval: 24,000 - 99,000). (B) Absolute number of glial cells in thousands plotted against the size (volume) of the piriform cortex. The regression line $\left(R^{2}=0.99\right.$, Coefficient of determination) is described by the equation: $N_{G}=43.5^{\star} V_{p o l}$, where $N_{G}$ is the number of glia (in thousands) and $\mathrm{Vol}_{p c x}$ is the piriform cortex volume in $\mathrm{mm}^{3}$ (95\% confidence interval: $\left.39,890-48,090\right)$. See Tables 1, S1-S3 for data values.

Next, we compared the total number of glial cells across species, and changes in the volume of piriform cortex. We found that the number of glial cells $\left(\mathrm{N}_{\mathrm{G}}\right)$ was proportional to the volume of the piriform cortex $\left(\mathrm{Vol}_{\mathrm{pcx}}, \mathrm{Fig} .2 \mathrm{~B}\right)$ and was an excellent fit for the equation $\mathrm{N}_{\mathrm{G}}=$ $m^{\star} \operatorname{Vol}_{p c x}\left(R^{2}=0.99, \mathrm{Cl}: 39.89-48.09\right)$, providing a second measure of glial volume density. Here, the slope $m=\mathrm{N}_{\mathrm{G}} / \mathrm{Vol}_{\mathrm{pcx}}$ is nothing but the volume density of glial cells and is $\mathrm{G}_{v}=43,500$ glia/mm $\mathrm{mm}^{3}$ (95\% confidence interval: $\left.39,890-48,090\right)$. For each glial cell that is added to the piriform cortex, neuropil volume is increased by an amount that covers $0.022 \mathrm{~nL}$ (1/volume density). 
Thus, an orderly relationship governs the distribution of glial cells across species: piriform cortex volume is proportional to glia number. The three analyses of our measurements yielded estimates of glia density that are not significantly different from each other, suggesting a common principle of glial organization in piriform cortices across mammalian species.

\section{Discussion}

One goal of the present study was to investigate whether a common principle of glial organization exists between phylogenetically divergent brain regions. Our data provides two pieces of evidence in support of such a common principle. First, the number of glial cells underneath a square $\mathrm{mm}$ scaled with the thickness of the piriform cortex, a major paleocortical structure, irrespective of the species examined. The analysis included comparisons of the piriform cortex from six species: mouse, rat, guinea pig, opossum, ferret, and cat. The overlap between species demonstrated in the scatter plot of Fig. 2B indicates that, just like the neocortex, the positive correlation between glial cells quantity and piriform cortex thickness does not depend on the species. Second, the total number of glial cells in the piriform cortex is proportional to the volume of the piriform cortex: an orderly relationship across species that suggests a common principle of glial organization. Taken together with previous evidence of a similar relationship in the neocortex (6), our work establishes an important conserved principle of glial organization across neocortices and paleocortices: the quantity of glia underneath $1 \mathrm{~mm}^{2}$ of surface increases systematically with cortical thickness, independently of the phylogenetic origin of the structures analyzed.

Although the relationship between glial cells and cortical thickness is similar in the neocortex and paleocortex, there is also an important difference: the volume occupied by a glial cell in the paleocortex is smaller. Indeed, for each glial cell that was added in the piriform cortex, neuropil volume was increased by an amount that covers $0.022 \mathrm{~nL}$. This is approximately half of what was found for the neocortex (6), and implies that the territory covered by a glial cell within 
this brain region corresponds to a cube with approximately $28 \mu \mathrm{m}$ per side, contrasting with 36 $40 \mu \mathrm{m}$ reported in the case of the neocortex $(7,8)$.

Two observations suggest that the greater density of glia in the paleocortex is to be expected. First, comparisons of neuronal and glial scaling in the neocortex (6) show that while glia increase with the thickness of the cortex, neurons do not, suggesting that glia cannot exclusively be needed for neuronal upkeep. Instead, it supports the assertions made earlier that the increase in glia and neuropil volume might reflect, in part, the territorial disposition of astrocytes, which have been postulated to support groups of synapses, axons, and dendrites (which would depend on cortical thickness) in an exclusive manner (9). Second, the organization of the connection matrix from the olfactory bulb to the piriform cortex in the mouse shows that every glomerulus contacts every piriform neuron (31). This all-to-all connectivity calls for a huge number of synapses, unlike the neocortex, where circuits are topographic and the number of synapses required might be fewer, as it is not an all-to-all network. Additionally, studies have shown that the molecular and deeper (layers I and III) layers of the piriform cortex are particularly synapse-rich (24). Measurements of glial surface density (Table S1) support these studies: across species, layers I and III have a greater number of glial cells than the piriform layer (layer II). Thus, it is likely that the synapse dense layers of the piriform cortex might require a higher number of astrocytes, in agreement with observations by (32).

In this report, we demonstrate that a conserved scaling law might explain the organization of glia in phylogenetically divergent structures in the brain: the neocortex and paleocortex. One limitation, however, is that our results do not yet demonstrate how differences in cell density might represent an adaptive advantage underlying the divergence of brain structures. Although such an objective was outside of the scope of the present study, future investigations are expected provide new insights into this exciting possibility. 


\section{Materials and Methods}

We used the same methods as earlier studies (6). Below, we present the stereological and histological procedures used. The numerical methods are briefly summarized in the results section and presented in more detail in the supplement, and in (33).

\section{Volumetric estimates}

The three piriform layers were outlined in successive coronal sections across the rostralcaudal extent with the help of Neurolucida (version 10.53; MBF Bioscience, Wilmington, VA) at low magnification (2X and $4 \mathrm{X}$ objectives) based on standard atlases and primary literature (25, 34). Once the layers from each individual section were outlined, all sections were aligned for subsequent three-dimensional reconstructions with Neurolucida explorer. Alignment was done by starting with the second section, and aligning each section to the previous one. Surface areas and volumes for the anterior piriform cortex were then obtained from three-dimensional reconstructions.

\section{Glial density}

10- or 20- $\mu$ m-wide columns, perpendicular to the pial surface, extending down to the boundary of layer 3, were delineated with the Neurolucida contours option. The sections and columns within them were chosen so that we had equal coverage along the rostral-caudal and dorsal-ventral extents, and equal amount of coverage of the APC. For obtaining surface area density counts, three measures were used: the width of the column, the thickness of the section, and the number of neurons in this column. Columns were randomly chosen while ensuring that they were close to perpendicular to our coronal cut and the surface of the brain (Table S4 for number of columns in each species). Glial cells were counted with standard unbiased stereology techniques in Nissl-stained sections at 100X oil magnification (Fig. 1C). The counting 
column functioned as a typical dissector whereby neurons and glia were marked as they come into focus given that they also fell within the acceptance lines of the dissector.

Glia were differentiated from neurons on the basis of size (smaller) and morphology (more punctate versus distinctive shape and processes extending out for neurons) of the cell, and by the presence of a nucleolus in neurons. Previous studies have shown that even though astrocytes have large cell bodies, in Nissl stained sections the cellular size that is stained is significantly smaller than neurons $(35,36)$. A few cells (less than $10 \%$ ) were hard to distinguish and were labeled as unknown and not included in the counts. Approximately 50 to 100 objects of interest were counted in each 3D column. We counted all the glia in our column from the pial surface to the white matter without the use of guard zones. A detailed discussion of guard zone use as it pertains to stereological methods and data collection for frozen sections is reported in (37).

\section{Nissl staining}

The tissue was defatted with $100 \%$ Ethanol: Chloroform (1:1) overnight, rehydrated in a decreasing alcohol (with $\mathrm{DI} \mathrm{H}_{2} \mathrm{O}$ ) series $\left(100,95,70\right.$, and $50 \%$ ), then treated with $\mathrm{DI} \mathrm{H}_{2} \mathrm{O}$, Thionin stain, followed by $\mathrm{DI} \mathrm{H}_{2} \mathrm{O}$, an increasing alcohol series $(50,70,95,95,100$, and 100 $\%)$, Xylenes I, Xylenes II, and then cover-slipped. The tissue was dipped 4-5 times in each of the solutions for 1 minute except for the thionin stain (1-2 minutes), and Xylenes II (1 hour). The thionin stain was prepared by first gently heating $1428 \mathrm{ml} \mathrm{DI} \mathrm{H} \mathrm{H}_{2} \mathrm{O}, 54 \mathrm{ml}$ of $1 \mathrm{M} \mathrm{NaOH}, 18 \mathrm{ml}$ of glacial acetic acid until the solution was steaming. Then, $3.75 \mathrm{~g}$ of thionin was added and the solution was boiled gently for $45 \mathrm{~min}$, cooled, filtered and used for staining.

\section{Histological procedures}

Brain tissues from healthy adult specimens belonging to the following six species: Mus musculus (mouse), Rattus novergicus (rat), Cavia porcellus (guinea pig), Mustela putoris furo 
(ferret), Monodelphis domestica (short-tailed opossum), and Felis catus (domestic cat), were analyzed. Animal care protocols were approved by the Salk Institute Animal and Use Committee and conform to US Department of Agriculture regulations and National Institutes of Health guidelines for humane care and use of laboratory animals. Each specimen was perfused with aldehyde fixative agents and stored long-term in $10 \%$ formalin. In preparation for cutting, all brains were submerged in solutions of $10 \%$ (wt/vol) glycerol and $10 \%$ formalin until the brain sank, and then moved into $20 \%$ glycerol and $10 \%$ formalin until the brain sank again; the average time in each solution was 3 to $10 \mathrm{~d}$. These cryoprotected brains were then cut coronally on a freezing microtome at a thickness of 40 or $50 \mu \mathrm{m}$. Every $6^{\text {th }}$ section was stained with thionin for visualization of Nissl bodies.

\section{Statistical analysis}

For the plots in Figures 1 and S1, we obtained the coefficient of determination using the R statistical programming language (38). We used inbuilt functions from $\mathrm{R}$ for calculating the best line fit, correlation coefficient, and the $95 \%$ confidence interval by fitting the data to a linear model. We have listed the correlation coefficient in the Figures and the results section of the main text.

For the density counts, assuming a homogenous spatial distribution, the column density counts should approximate a Gaussian distribution. This happens because with a spatially homogenous distribution, as one traverses a line from a fixed point, one will encounter glial cells. The probability of encountering a glial cell is given by a Poisson distribution in this case, as their distribution is homogenous. As a result, in the limit, with a large number of cells, the distribution of densities of different columns will follow a Gaussian distribution. We validated our column counts by fitting a relative cumulative distribution to a cumulative Gaussian and by verifying that the fit was adequate. 


\section{References}

1. C. F. Stevens, An evolutionary scaling law for the primate visual system and its basis in cortical function. Nature. 411, 193-195 (2001).

2. B. L. Finlay, R. B. Darlington, Linked regularities in the development and evolution of mammalian brains. Science. 268, 1578-84 (1995).

3. H. Cuntz, A. Mathy, M. Häusser, A scaling law derived from optimal dendritic wiring. Proc. Natl. Acad. Sci. U. S. A. 109, 11014-8 (2012).

4. G. Buzsáki et al., Scaling Brain Size, Keeping Timing: Evolutionary Preservation of Brain Rhythms. Neuron. 80, 751-764 (2013).

5. N. J. Allen, B. A. Barres, Neuroscience: Glia - more than just brain glue. Nature. 457, 675-677 (2009).

6. C. N. Carlo, C. F. Stevens, Structural uniformity of neocortex, revisited. Proc. Natl. Acad. Sci. U. S. A. 110, 1488-93 (2013).

7. K. Ogata, T. Kosaka, Structural and quantitative analysis of astrocytes in the mouse hippocampus. Neuroscience. 113, 221-33 (2002).

8. E. A. Bushong, M. E. Martone, Y. Z. Jones, M. H. Ellisman, Protoplasmic astrocytes in CA1 stratum radiatum occupy separate anatomical domains. J. Neurosci. 22, 183-92 (2002).

9. M. M. Halassa, T. Fellin, H. Takano, J.-H. Dong, P. G. Haydon, Synaptic Islands Defined by the Territory of a Single Astrocyte. J. Neurosci. 27, 6473-6477 (2007).

10. N. J. Abbott, L. Rönnbäck, E. Hansson, Astrocyte-endothelial interactions at the bloodbrain barrier. Nat. Rev. Neurosci. 7, 41-53 (2006).

11. P. Kofuji, E. A. Newman, Potassium buffering in the central nervous system. Neuroscience. 129, 1045-56 (2004).

12. D. E. Bergles, C. E. Jahr, Synaptic activation of glutamate transporters in hippocampal 
astrocytes. Neuron. 19, 1297-308 (1997).

13. J. S. Diamond, C. E. Jahr, Synaptically Released Glutamate Does Not Overwhelm Transporters on Hippocampal Astrocytes During High-Frequency Stimulation. J. Neurophysiol. 83 (2000).

14. N. C. Danbolt, Glutamate uptake. Prog. Neurobiol. 65, 1-105 (2001).

15. J. S. Diamond, Deriving the Glutamate Clearance Time Course from Transporter Currents in CA1 Hippocampal Astrocytes: Transmitter Uptake Gets Faster during Development. J. Neurosci. 25 (2005).

16. M. Bélanger et al., Brain Energy Metabolism: Focus on Astrocyte-Neuron Metabolic Cooperation. Cell Metab. 14, 724-738 (2011).

17. A. Panatier et al., Astrocytes are endogenous regulators of basal transmission at central synapses. Cell. 146, 785-798.

18. G. Perea, A. Araque, Astrocytes potentiate transmitter release at single hippocampal synapses. Science. 317, 1083-6 (2007).

19. Y. Yang et al., Contribution of astrocytes to hippocampal long-term potentiation through release of D-serine. Proc. Natl. Acad. Sci. U. S. A. 100, 15194-9 (2003).

20. C. Henneberger, T. Papouin, S. H. R. Oliet, D. A. Rusakov, Long-term potentiation depends on release of d-serine from astrocytes. Nature. 463, 232-236 (2010).

21. T. Sasaki et al., Astrocyte calcium signalling orchestrates neuronal synchronization in organotypic hippocampal slices. J. Physiol. 592, 2771-83 (2014).

22. J. Foley et al., Astrocytic IP3/Ca(2+) Signaling Modulates Theta Rhythm and REM Sleep. Front. Neural Circuits. 11, 3 (2017).

23. H. S. Lee et al., Astrocytes contribute to gamma oscillations and recognition memory. Proc. Natl. Acad. Sci. 111, E3343-E3352 (2014).

24. L. B. Haberly, Olfactory cortex. (1998).

25. K. R. Neville, L. B. Haberly, Olfactory cortex. synaptic Organ. brain. 5, 415-454 (2004). 
26. J. M. Bekkers, N. Suzuki, Neurons and circuits for odor processing in the piriform cortex. Trends Neurosci. 36, 429-438 (2013).

27. D. H. Hubel, T. N. Wiesel, Receptive fields, binocular interaction and functional architecture in the cat's visual cortex. J. Physiol. 160, 106 (1962).

28. D. D. Stettler, R. Axel, Representations of Odor in the Piriform Cortex. Neuron. 63, 854864 (2009).

29. K. R. Illig, L. B. Haberly, Odor-evoked activity is spatially distributed in piriform cortex. J. Comp. Neurol. 457, 361-373 (2003).

30. W. Yang, Q.-Q. Sun, Hierarchical organization of long-range circuits in the olfactory cortices. Physiol. Rep. 3 (2015), doi:10.14814/phy2.12550.

31. S. Srinivasan, C. F. Stevens, The distributed circuit in the piriform cortex makes odor discrimination robust. J. Comp. Neurol. (2018).

32. U. Faraguna, A. Nelson, V. V Vyazovskiy, C. Cirelli, G. Tononi, Unilateral cortical spreading depression affects sleep need and induces molecular and electrophysiological signs of synaptic potentiation in vivo. Cereb. cortex. 20, 2939-2947 (2010).

33. S. Srinivasan, C. F. Stevens, A quantitative description of the mouse piriform cortex. bioRxiv, 99002 (2017).

34. G. Paxinos, K. B. J. Franklin, The mouse brain in stereotaxic coordinates (Gulf Professional Publishing, 2004).

35. H. J. Jyothi et al., Aging causes morphological alterations in astrocytes and microglia in human substantia nigra pars compacta. Neurobiol. Aging. 36, 3321-3333 (2015).

36. M. Á. García-Cabezas, Y. J. John, H. Barbas, B. Zikopoulos, Distinction of Neurons, Glia and Endothelial Cells in the Cerebral Cortex: An Algorithm Based on Cytological Features. Front. Neuroanat. 10, 107 (2016).

37. C. N. Carlo, C. F. Stevens, Analysis of differential shrinkage in frozen brain sections and its implications for the use of guard zones in stereology. J Comp Neurol. 519, 2803-2810 
bioRxiv preprint doi: https://doi.org/10.1101/449421; this version posted October 29, 2019. The copyright holder for this preprint (which was not certified by peer review) is the author/funder, who has granted bioRxiv a license to display the preprint in perpetuity. It is made available under aCC-BY-NC-ND 4.0 International license.

(2011).

38. R Core Team, R: A Language and Environment for Statistical Computing (2013), (available at http://www.r-project.org/). 
Acknowledgements: We are grateful to Chuck Stevens, whose work on the neocortex inspired this study, for his feedback and encouragement. We are also grateful to the Kavli Institute for Brain and Mind at the University of California, San Diego for funding APD, SS, and TJS through the Innovative Research Grants program.

Author contributions: SS, KB, and APD designed the study, performed and analyzed experiments, APD and SS interpreted the data and wrote the manuscript, and TJS provided critical feedback. All authors revised and approved the manuscript.

Competing interests: The authors declare that they have no competing interests.

Data and materials availability: Data is available upon request. 
Table 1. Data points plotted in Figures 1 and 2. Averages are given for animals with more than one specimen. Values for individual specimens of each species are provided in Tables S1 and S2.

\begin{tabular}{|l|l|l|l|l|l|l|}
\hline $\begin{array}{l}\text { Common } \\
\text { name }\end{array}$ & $\begin{array}{l}\text { Brain } \\
\text { volume } \\
\left(\mathrm{cm}^{3}\right)\end{array}$ & $\begin{array}{l}\text { APC } \\
\text { surface } \\
\text { area }\left(\mathrm{mm}^{2}\right)\end{array}$ & $\begin{array}{l}\text { APC } \\
\text { Volume } \\
\left(\mathrm{mm}^{3}\right)\end{array}$ & $\begin{array}{l}\text { APC width } \\
(\mathrm{mm})\end{array}$ & $\begin{array}{l}\text { Surface } \\
\text { density } \\
(\mathrm{x} \mathrm{1000} \\
\left.\mathrm{glia} / \mathrm{mm}^{2}\right)\end{array}$ & $\begin{array}{l}\text { Number of } \\
\text { glia } \\
(\mathrm{x} \mathrm{1000)}\end{array}$ \\
\hline Cat & 33 & 58.0 & 49.9 & 0.88 & 37.5 & 2175 \\
\hline Ferret & 10 & 19.4 & 13.2 & 0.69 & 31.5 & 611 \\
\hline Guinea pig & 3.9 & 19.8 & 17.2 & 0.85 & 43.5 & 862 \\
\hline Mouse & 0.3 & 4.7 & 2.6 & 0.55 & 18.0 & 85 \\
\hline Opossum & 1 & 9.5 & 8.3 & 0.86 & 37.4 & 355 \\
\hline Rat & 1.7 & 10.7 & 7.2 & 0.68 & 28.0 & 298 \\
\hline
\end{tabular}




\section{Supplementary materials}
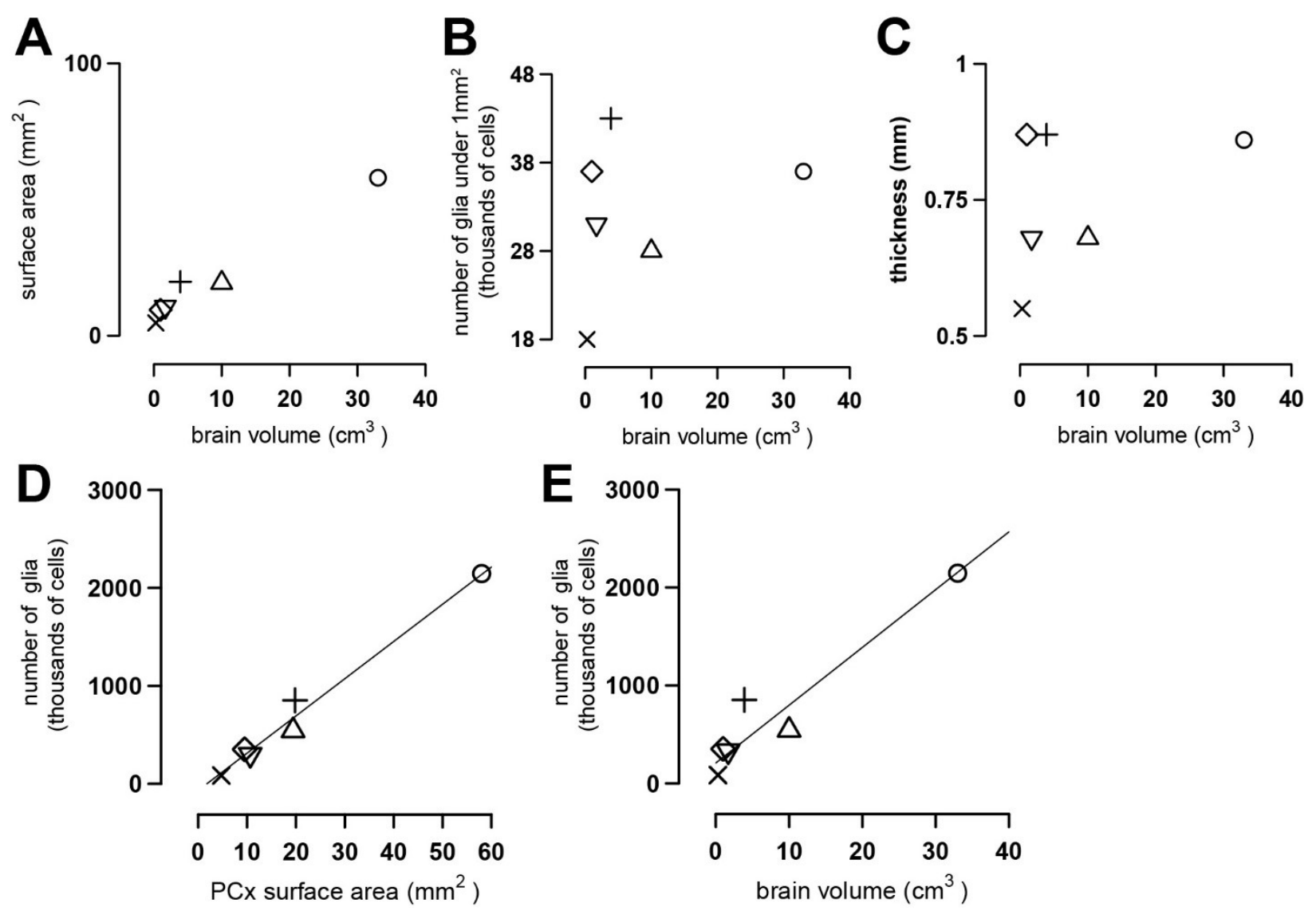

Fig. S1. Piriform cortex measurements across species. The number of glial cells was plotted for six species of mammals: mouse $(\times)$, rat $(\nabla)$, ferret $(\Delta)$, opossum $(\diamond)$, cat $(0)$, guinea pig $(+)$. (A) The surface area of the piriform cortex increases with an increase in brain volume $\left(\mathrm{cm}^{3}\right)$. (B) The number of glia underneath a square $\mathrm{mm}$ versus brain volume. (C) The thickness of the piriform cortex versus brain volume. (D) Absolute number of glial cells in thousands plotted against the size (surface area) of the piriform cortex $\left(R^{2}=0.98\right.$, Coefficient of determination). The regression line is described by the equation: $y=-67+38^{*} x$, where $y$ is the number of glia (in thousands) and $x$ is the piriform cortex surface area in $\mathrm{mm}^{2}$. (E) Absolute number of glial cells in thousands plotted against the size (volume) of the brain $\left(R^{2}=0.86\right.$, Coefficient of determination). The regression line is described by the equation: $y=-242+59^{*} x$, where $y$ is the number of glia (in thousands) and $x$ is the brain volume in $\mathrm{cm}^{3}$. 
Table S1. The surface area density (the number of glia underneath a square $\mathrm{mm}$ ) of the overall Piriform cortex. We also present results for individual layers (note that in this case, cells located on the border of two contiguous layers have been counted in both of those layers).

\begin{tabular}{|l|l|l|l|l|}
\hline Common name & Overall density $\left(\mathrm{glia} / \mathrm{mm}^{2}\right)$ & Layer 1 & Layer 2 & Layer 3 \\
\hline Cat & 37507.20 & 17280.30 & 4977.55 & 15249.67 \\
\hline Ferret I & 27606.77 & 11821.10 & 4226.61 & 11559.05 \\
\hline Ferret II & 36708.36 & 10293.71 & 13671.75 & 14158.78 \\
\hline Guinea pig I & 43942.16 & 15581.59 & 11108.65 & 17251.92 \\
\hline Guinea pig II & 43522.39 & 14834.07 & 13870.22 & 14818.10 \\
\hline Mouse I & 18517.28 & 8378.69 & 4415.78 & 5722.79 \\
\hline Mouse II & 20393.09 & 7968.30 & 5414.40 & 7010.37 \\
\hline Opossum & 37427.59 & 11610.04 & 8660.42 & 18424.03 \\
\hline Rat I & 31788.16 & 17722.54 & 3275.22 & 10998.75 \\
\hline Rat II & 24005.74 & 12963.53 & 3250.73 & 7963.00 \\
\hline
\end{tabular}

Table S2. The width of the overall Piriform cortex and its individual layers

\begin{tabular}{|l|l|l|l|l|}
\hline Common name & Overall width $(\mathrm{mm})$ & Layer 1 & Layer 2 & Layer 3 \\
\hline Cat & 0.8793 & 0.2744 & 0.1971 & 0.4077 \\
\hline Ferret I & 0.6630 & 0.2248 & 0.2225 & 0.2194 \\
\hline Ferret II & 0.70666 & 0.2422 & 0.2082 & 0.2561 \\
\hline Guinea pig I & 0.8760 & 0.2723 & 0.3023 & 0.3012 \\
\hline Guinea pig II & 0.8151 & 0.2500 & 0.2924 & 0.2727 \\
\hline Mouse I & 0.5553 & 0.1761 & 0.1988 & 0.1813 \\
\hline Mouse II & 0.5266 & 0.1683 & 0.1656 & 0.1968 \\
\hline
\end{tabular}




\begin{tabular}{|l|l|l|l|l|}
\hline Opossum & 0.8639 & 0.2545 & 0.1605 & 0.4838 \\
\hline Rat I & 0.7194 & 0.2482 & 0.1490 & 0.3121 \\
\hline Rat II & 0.6483 & 0.2453 & 0.1805 & 0.2252 \\
\hline
\end{tabular}

Table S3. Glial cells volume density.

\begin{tabular}{|l|l|}
\hline Common name & Vol. density (glial cells/mm ${ }^{3}$ ) \\
\hline Cat & 42,660 \\
\hline Ferret I & 41,598 \\
\hline Ferret II & 52,066 \\
\hline Guinea Pig I & 49,800 \\
\hline Guinea Pig II & 56,000 \\
\hline Mouse I & 34,020 \\
\hline Mouse II & 40,693 \\
\hline Opossum & 43,800 \\
\hline Rat I & 45,000 \\
\hline Rat II & 36,861 \\
\hline Mean \pm SEM & $44,249 \pm 2,030$ \\
\hline
\end{tabular}

Table S4. Number of cortical columns analyzed per animal.

\begin{tabular}{|l|l|}
\hline Common name & \# of columns \\
\hline
\end{tabular}


bioRxiv preprint doi: https://doi.org/10.1101/449421; this version posted October 29, 2019. The copyright holder for this preprint (which was not certified by peer review) is the author/funder, who has granted bioRxiv a license to display the preprint in perpetuity. It is made available under aCC-BY-NC-ND 4.0 International license.

\begin{tabular}{|l|l|}
\hline Cat & 10 \\
\hline Ferret I & 16 \\
\hline Ferret II & 10 \\
\hline Guinea Pig I & 11 \\
\hline Guinea Pig II & 9 \\
\hline Mouse I & 24 \\
\hline Mouse II & 8 \\
\hline Opossum & 16 \\
\hline Rat I & 15 \\
\hline Rat II & 21 \\
\hline Mean \pm SEM & $14 \pm 1.7$ \\
\hline
\end{tabular}

